\title{
On the Conditional Behavior of Stock Market Volatility: A Sub-Sample Analysis Using the FIGARCH Approach for Developed and Emerging Markets
}

\begin{abstract}
S.R. BENTES*
Instituto Superior de Contabilidade e Administração de Lisboa (ISCAL), Lisboa, Portugal

and Instituto Universitário de Lisboa (ISCTE-IUL), Business Research Unit (BRU-IUL), Lisboa, Portugal

Long memory has always played a central role in physics since it was first discovered by Hurst while studying the flow of the River Nile. Interestingly, after his seminal work, many other researchers found the same pattern in other domains of science, such as biology, economics and finance. These studies have mainly relied on the use of the Hurst exponents as a measure of the degree of memory in a process. In this paper we use a different approach based on the FIGARCH (fractional integrated generalized autoregressive conditionally heteroskedasticity) model proposed by Baillie et al. in order to analyze the long memory behavior of stock market volatility. More specifically, we compare how the long memory parameter evolves before and after the 2008 and 2012 crises in both developed and emerging markets. Specifically, we consider the daily returns of the S\&P 500, STOXX 50, FTSE 100, NIKKEI 225, HSI, BUX, WIG, SSE, IDX and KLCI indices for the period from October 1, 2003 to October 2, 2015 and then split the whole sample into four sub-samples of roughly three years each. Results show different patterns for the pre and post crisis periods revealing that the degree of memory differs in accordance with the country's development and the level of market turbulence. In particular, we found that major mature economies present higher levels of long memory than emerging countries and were more affected by the 2008 and 2012 crises.
\end{abstract}

DOI: 10.12693/APhysPolA.129.997

PACS/topics: 89.65.Gh

\section{Introduction}

Volatility is a prevailing characteristic of stock markets that has major consequences in financial activities such as risk management, investment decisions and portfolio valuation, to cite just a few. Thus, understanding its implications is crucial to accurately assess investment risk and develop trading strategies. One important feature that characterizes volatility is long memory. Originally dicovered by Hurst [1] in the domain of hydrology, this concept was rapidly extended to other domains of science such as geophysics, biology, etc. According to this author, who derived the so-called Hurst exponent, two different situations could occur while analyzing the degree of memory in a process: (a) for $H=1 / 2$ we have the usual Brownian motion; but (b) for $H \neq 1 / 2$ there is evidence of long range correlations. For an overview of the various estimators of the Hurst exponents such as rescaled range analysis $(\mathrm{R} / \mathrm{S})$ and detrended fluctuation analysis (DFA) refer to Taqqu et al. [2].

The first known attempt to use this concept in finance is found in Mandelbrot and van Ness [3]. Since then, many other studies have applied this method to describe price dynamics (e.g., Refs. [4-7]). A Hurst exponent greater than $1 / 2$ is usually found, which means that financial time series generally exhibit some degree of memory. The major consequence of this is that shocks to the volatility process tend to linger for some time as volatility

*e-mail: smbentes@iscal.ipl.pt displays little tendency to revert toward its mean. Accordingly, the potential predictability of time series may lead to the invalidity of the weak form of the efficient market hypothesis [8].

In our study, we apply an alternative approach based on the $\operatorname{FIGARCH}(p, d, q)$ model (fractional integrated GARCH) introduced by Baillie et al. [9] to capture the long memory behavior of a time series. The great innovation of this method is that it considers the temporal variation of volatility. It also generalizes GARCH and the integrated GARCH (IGARCH) formulations, which have proved unsuitable to capture this feature of the data. In fact, the GARCH model only accounts for short memory, while the IGARCH considers infinite memory, which is a very unrealistic situation. By introducing a fractional differencing parameter $d$, the FIGARCH model allows long memory in the observed data to be accounted for as $0<d<1$ and accommodates both the GARCH $(d=0)$ and the IGARCH $(d=1)$ frameworks as special cases.

This paper adds to the literature by examining how the fractional differencing parameter $d$ evolves before and after the 2008 and 2012 financial crises for emerging and developed countries. To this end, we gathered price index data from October 1, 2003 to October 2, 2015 for five emerging countries (BUX, WIG, SSE, IDX and KLCI) and for five developed markets (S\&P 500, STOXX 50, FTSE 100, NIKKEI 225, HSI) and estimate the FIGARCH model for the full sample period. We then split the whole sample into four sub-samples of roughly three years each that encompass stable and crisis periods.

Foreshadowing our main results, we find evidence that 
shocks have long memory effects on volatility and that these effects are more pronounced in developed than emerging countries. Moreover, developed countries registered more memory effects during both financial crises than the emerging countries.

We organize the remainder of the paper as follows. We discuss the econometric methodology in Sect. 2. The empirical analysis is presented in Sect. 3. Section 4 concludes.

\section{Econometric methodology}

\subsection{GARCH model}

Following Engle [10], we consider the time series $y_{t}$ and the associated prediction error $\varepsilon_{t}=y_{t}-E_{t-1}\left[y_{t}\right]$, where $E_{t-1}[$.$] is the expectation or the conditional mean$ on the information set at time $t-1$. The symmetric $\operatorname{GARCH}(p, q)$ model introduced by Bollerslev [11] is defined as:

$$
\sigma_{t}^{2}=\omega+\alpha(L) \varepsilon_{t}^{2}+\beta(L) \sigma_{t}^{2},
$$

where $\omega>0, \alpha \geq 0, \beta \geq 0, L$ denotes the lag or backshift operator $\alpha(L) \equiv \alpha_{1} L+\alpha_{2} L^{2}+\ldots+\alpha_{q} L^{q}$ and $\beta(L) \equiv \beta_{1} L+\beta_{2} L^{2}+\ldots+\beta_{p} L^{p}$. More specifically, the $\operatorname{GARCH}(1,1)$ model generates volatility forecasts as a weighted average of the constant or average variance, the previous forecasting variance and the previous volatility, thus reflecting the squared news about the returns. In addition, the $\operatorname{GARCH}(p, q)$ framework presents some major advantages over the seminal $\operatorname{ARCH}(q)$ model of Engle [10]: (i) it is more parsimonious and (ii) avoids overfitting. Consequently, it is less prone to breach nonnegativity constraints than the standard $\operatorname{ARCH}(q)$.

It should be noted that the apparent long memory implied by the estimates of the conditional variance is a common empirical finding in applied work. This is manifested by the presence of an approximate unit root in the autoregressive polynomial, i.e. $\alpha+\beta \approx 1$ [12]. Since the $\operatorname{GARCH}(p, q)$ formulation considers that shocks decay at a fast geometric rate, this specification is only suitable to describe short-memory phenomena and not for long memory.

\subsection{IGARCH model}

In order to overcome the above-mentioned limitation, Engle and Bollerslev [13] introduced the $\operatorname{IGARCH}(p, q)$ model, which captures $I(1)$ type processes for the conditional variance as infinite long memory remains important for forecasts of all horizons. Assuming that $v_{t} \equiv \varepsilon_{t}^{2}-\sigma_{t}^{2}$ the $\operatorname{GARCH}(p, q)$ model can be re-written in the form of an $\operatorname{ARMA}(m, p)$ process

$$
\Phi(L)(1-L) \varepsilon_{t}^{2}=\omega+[1-\beta(L)] v_{t},
$$

where $\Phi(L)=[1-\alpha(L)-\beta(L)](1-L)^{-1}$ and all roots of $\Phi(L)$ and $[1-\beta(L)]$ lie outside the unit root circle.

Although an improvement over its predecessor, this model is not entirely satisfactory when describing long memory in the volatility process because it considers that shocks never die out. Thus, a model which accounts for an intermediate range of memory seems to be necessary.

\subsection{FIGARCH model}

In an attempt to describe the long memory process of a time series more realistically Baillie et al. [9] introduced a new class of models called $\operatorname{FIGARCH}(p, d, q)$. In contrast to an $I(0)$ time series in which shocks die out at a fast geometric rate or an $I(1)$ time series where there is no mean reversion, shocks to an $I(d)$ time series with $0 \leq$ $d \leq 1$ decay at a very slow hyperbolic rate.

The $\operatorname{FIGARCH}(p, d, q)$ model can be obtained by replacing the differencing operator in Eq. (2) with a fractional differencing operator $(1-L)^{d}$ as in the following:

$$
\Phi(L)(1-L)^{d} \varepsilon_{t}^{2}=\omega+[1-\beta(L)] v_{t} .
$$

Rearranging the terms in Eq. (3), the $\operatorname{FIGARCH}(p, d, q)$ model can be written as follows:

$$
[1-\beta(L)] \sigma_{t}^{2}=\omega+\left[1-\beta(L)-\Phi(L)(1-L)^{d}\right] \varepsilon_{t}^{2} .
$$

The conditional variance of $\varepsilon_{t}^{2}$ is obtained by

$$
\sigma_{t}^{2}=\frac{\omega}{[1-\beta(L)]}+\left[1-\frac{\Phi(L)}{[1-\beta(L)]}(1-L)^{d}\right] \varepsilon_{t}^{2},
$$

which corresponds to

$$
\sigma_{t}^{2}=\frac{\omega}{[1-\beta(L)]}+\lambda(L) \varepsilon_{t}^{2},
$$

where $\lambda(L)=\lambda_{1} L+\lambda_{2} L^{2} \ldots$

The major advantage of the $\operatorname{FIGARCH}(p, d, q)$ approach compared over the aforementioned methods is that it provides greater flexibility for modeling the conditional variance since it accommodates the covariance stationary $\operatorname{GARCH}(p, q)$ model when $d=0$ and the $\operatorname{IGARCH}(p, q)$ model when $d=1$, as special cases.

For the $\operatorname{FIGARCH}(p, d, q)$ process, the long memory property of shocks to the conditional variance is measured by the fractional differencing parameter $d$. Hence, the attraction of this methodology is that for $0 \leq d \leq 1$, it is sufficiently flexible to allow for an intermediate range of long memory because shocks in the variance decrease at a very slow hyperbolic rate of decay. Notice that the parameters in the $\operatorname{FIGARCH}(p, d, q)$ method can be estimated by an approximate quasi-maximum likelihood estimation technique, as advocated by Bollerslev and Wooldridge [14].

\section{Empirical analysis}

\subsection{Data}

We use daily continuously compounded returns of ten stock market indices in order to compare the long memory behavior of stock market volatility in developed and emerging economies. Using the 2014 MSCI market classification list to distinguish between developed and emerging markets, we then selected five indices for each classification. More specifically, we chose the S\&P 500 (US), STOXX 50 (EU), FTSE 100 (UK), NIKKEI 225 (Japan) and HSI (Hong Kong) indices for the group of developed markets and the BUX (Hungary), WIG (Poland), SSE AS (China), IDX (Indonesia) and KLCI (Malaysia) indices for the set of emerging markets.

The overall sample period covers 12 years from October 1, 2003 to October 2, 2015, providing 3130 daily 
returns for each series. Since we intend to analyze how the parameter $d$ evolves in different economic cycles, we divided the full sample period into four sub-samples of three years each, as follows: (i) October 6, 2003 - September 29, 2006; (ii) October 2, 2006 - October 2, 2009; (iii) October 5, 2009 - September 28, 2012, and (iv) October 1, 2012 - October 2, 2015. This allows us to examine the variation in the degree of long memory across countries and across stable/crisis periods. All series were converted to the base 100, which we set at the beginning of the sample period (October 6, 2003) for comparative purposes. The data were sourced from DataStream International.

Figures 1 and 2 illustrate the price evolution of the ten market indices. Despite some similarities between the two figures given that peaks and troughs seem to occur at the same time, prices in emerging markets exhibit a much more unsteady behavior that in developed markets. Indeed, whereas there are small variations in developed markets' indices with NIKKEI 225 and FTSE 100 evidencing the smallest fluctuations, the indices in emerging markets display changes of a greater magnitude.

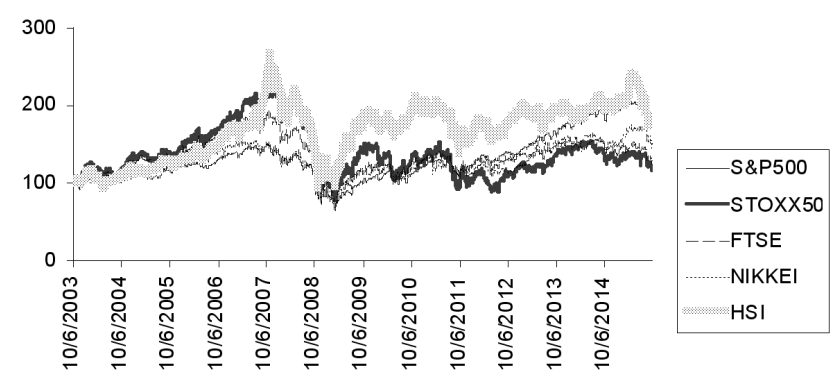

Fig. 1. Daily closing prices of the developed countries indices.

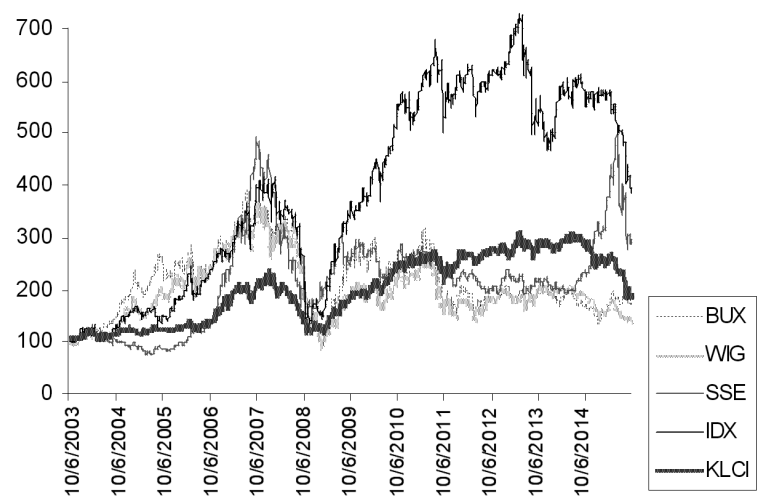

Fig. 2. Daily closing prices of the emerging countries indices.

Tables I and II present the summary statistics, heteroskedasticity and unit root tests of the daily returns for the whole sample period. The descriptive statistics are reported in panel A. Results show that all returns exhibit a very low mean when compared with the standard deviation. The average daily returns are found to be positive with the standard deviation varying from around 0.01 for KLCI and around 0.02 for BUX. Notably, the higher standard deviations in the emerging countries clearly illustrate the higher levels of volatility typically associated with these markets, which are usually regarded as riskier than the mature economies.

In general, there is also evidence of negative skewness; however, HSI is skewed toward the right thus revealing that large positive stock returns were more common than large negative returns for this index. The reverse situation was found in the remaining indices, therefore showing the striking impact of negative returns. Another important feature of the data is the high level of kurtosis, which ranges from 7.16 for SSE to 15.1142 for S\&P 500 indicating a fatter tailed distribution than the Normal. This is further corroborated by the JarqueBera test, which rejects the null hypothesis of Gaussianity at the $1 \%$ level for all cases. Moreover, both the Ljung-Box $Q$ test statistic with 10 lags $(Q(10))$ and the Breusch-Godfrey Lagrange multiplier test statistics with 10-period lag $(\mathrm{LM}(10))$ reveal the presence of serial correlation in all series. There is also evidence of heteroskedasticity as given by $\mathrm{ARCH}-\mathrm{LM}(10)$ and $Q^{2}(10)$ test statistics displayed in Panel $\mathrm{B}$ of both tables. These tests were employed to double check the presence of ARCH effects and postulate the null hypothesis that the series is homocedastic. It should be noted that there was no specific reason for choosing a 10-period lag as the test statistics were all significant at the $1 \%$ level for $q=1,2, \ldots, 10$. This is true for all the above-mentioned tests.

Finally, panel C of Tables I and II shows the augmented Dickey Fuller (ADF) and the Kwiatkowski, Phillips, Schmidt and Shin (KPSS) unit root test statistics. While the ADF test postulates the null hypothesis that the series has a unit root, the KPSS considers the exact opposite i.e., that the series is stationary. Results reveal that all series are stationary as required for further analysis of the data.

\subsection{Estimation results}

Since all return series reveal serial correlation we fit an autoregressive model to remove it from the data. Based on the correlograms we chose an $\mathrm{AR}(5)$ specification for all series. Next, we perform a battery of tests to investigate the adequacy of a time series model to describe the data. More specifically, we employ the Ljung-Box $Q$ and the Breusch-Godfrey Lagrange multiplier test to check for autocorrelation as well as the Lagrange multiplier test of Engle [10] and the Ljung-Box $Q$ test of the squared residuals $\left(Q^{2}(10)\right)$ to search for heteroskedasticity. Results show that the specified $\operatorname{AR}(p)$ models were sufficient to capture any autocorrelation present in the data; however heteroskedasticity still persists, thus favoring the use of GARCH-type models. These results are not reported here to save space but are available from the author upon request. 


\section{TABLE I}

Preliminary analysis of stock market daily returns in developed countries. J-B - the statistics of the Jarque and Bera normal distribution test. $Q(n)$ and $Q^{2}(n)$ - the Ljung-Box statistics testing for serial correlation of the return series and the squared returns, respectively, up to the $n$-th order for 10 lags in both situations. LM(10) - Breusch-Godfrey test for the null hypothesis of no serial correlation up to 10 lags. ARCH-LM - ARCH test for the null of no autoregressive conditional heteroskedasticity up to 10 lags. ADF - augmented Dickey and Fuller test for the null of non-stationarity. Mackinnon et al. [15] critical values: $-3.43(1 \%)$ and $-2.86(5 \%)$ for constant and $-3.96(1 \%)$ and $-3.41(5 \%)$ for constant and linear trend. KPSS - the Kwiatkowski, Phillips, Schmidt and Shin test for the null of stationarity. Critical values: $0.739(1 \%)$ and $0.463(5 \%)$ for constant and $0.216(1 \%)$ and $0.146(5 \%)$ for constant and linear trend. $\dagger$ indicates a rejection of the null hypothesis at the $1 \%$ significance level.

\begin{tabular}{c|c|c|c|c|c}
\hline \hline & S\&P500 & STOXX 50 & FTSE 100 & NIKKEI 225 & HSI \\
\hline \multicolumn{7}{c}{ A - summary statistics } \\
\hline mean & 0.0002 & $5.56 \times 10^{-5}$ & $8.57 \times 10^{-5}$ & 0.0001 & 0.0002 \\
sd & 0.012 & 0.0164 & 0.0141 & 0.0146 & 0.015 \\
skewness & -0.3428 & -0.0215 & -0.1374 & -0.364 & 0.0184 \\
kustosis & 15.1142 & 9.5481 & 13.3419 & 8.9364 & 13.0827 \\
J-B [16] & $19200.3^{\dagger}$ & $5592.14^{\dagger}$ & $13958.5^{\dagger}$ & $4665.09^{\dagger}$ & $13258.5^{\dagger}$ \\
$Q(10)$ & $60.431^{\dagger}$ & $42.263^{\dagger}$ & $63.884^{\dagger}$ & $61.457^{\dagger}$ & $41.807^{\dagger}$ \\
LM(10) & $6.2273^{\dagger}$ & $4.0359^{\dagger}$ & $6.2835^{\dagger}$ & $7.0659^{\dagger}$ & $2.8821^{\dagger}$ \\
\hline \multicolumn{7}{c}{} \\
\hline ARCH-LM(10) & $126.667^{\dagger}$ & $72.4728^{\dagger}$ & $113.915^{\dagger}$ & $96.1169^{\dagger}$ & $115.999^{\dagger}$ \\
$Q^{2}(10)$ & $2855^{\dagger}$ & $1585.3^{\dagger}$ & $2380.4^{\dagger}$ & $61.457^{\dagger}$ & $2363.9^{\dagger}$ \\
\hline ADF [17] & $-43.9723^{\dagger}$ & $-57.5743^{\dagger}$ & $-27.1035^{\dagger}$ & $-44.8281^{\dagger}$ & $-57.3293^{\dagger}$ \\
KPSS [18] & 0.0848 & 0.0651 & 0.0773 & 0.0525 & 0.0318
\end{tabular}

TABLE II

Preliminary analysis of stock market daily returns in emerging countries. J-B - the statistics of the Jarque and Bera normal distribution test. $Q(n)$ and $Q^{2}(n)$ - the Ljung-Box statistics testing for autocorrelation in the standardized residuals and squared standardized residuals up to the 10th lag respectively. The latter is used to check for ARCH effects while the former is employed to test for serial dependence. LM(10) - Breusch-Godfrey test for the null hypothesis of no serial correlation up to 10 lags. ARCH-LM - ARCH test for the null of no autoregressive conditional heteroskedasticity up to 10 lags. ADF - augmented Dickey and Fuller test for the null of non-stationarity. Mackinnon et al. [15] critical values: $-3.43(1 \%)$ and $-2.86(5 \%)$ for constant and $-3.96(1 \%)$ and $-3.41(5 \%)$ for constant and linear trend. KPSS the Kwiatkowski, Phillips, Schmidt and Shin test for the null of stationarity. Critical values: $0.739(1 \%)$ and $0.463(5 \%)$ for constant and $0.216(1 \%)$ and $0.146(5 \%)$ for constant and linear trend. $\dagger$ significance level: $1 \%$.

\begin{tabular}{c|c|c|c|c|c}
\hline \hline & BUX & WIG & SSE & IDX & KLCI \\
\hline \multicolumn{7}{|c|}{ A - summary statistics } \\
\hline mean & 0.0002 & 0.0001 & 0.0003 & 0.0004 & 0.0002 \\
sd & 0.0215 & 0.0202 & 0.0167 & 0.0164 & 0.0094 \\
skewness & -0.0314 & -0.2526 & -0.4576 & -0.5722 & -0.6499 \\
kustosis & 10.6444 & 7.8893 & 7.16 & 11.3842 & 11.9758 \\
J-B [16] & $7621.68^{\dagger}$ & $3150.88^{\dagger}$ & $2366.16^{\dagger}$ & $9338.42^{\dagger}$ & $10727.4^{\dagger}$ \\
$Q(10)$ & $62.393^{\dagger}$ & $10.94^{\dagger}$ & $33.85^{\dagger}$ & $46.321^{\dagger}$ & $55.394^{\dagger}$ \\
LM(10) & $5.6624^{\dagger}$ & $5.6042^{\dagger}$ & $3.1341^{\dagger}$ & $4.8097^{\dagger}$ & $5.5034^{\dagger}$ \\
\hline \multicolumn{7}{|c|}{ B - heteroskedasticity tests } \\
\hline ARCH-LM(10) & $72.4835^{\dagger}$ & $64.8362^{\dagger}$ & $32.5974^{\dagger}$ & $31.8387^{\dagger}$ & $16.5901^{\dagger}$ \\
$Q^{2}(10)$ & $1520.9^{\dagger}$ & $1305.2^{\dagger}$ & $632.66^{\dagger}$ & $623.07^{\dagger}$ & $260.8^{\dagger}$ \\
\hline ADF [17] & $-40.6594^{\dagger}$ & $-52.776^{\dagger}$ & $-54.7872^{\dagger}$ & $-50.58^{\dagger}$ & $-50.0514^{\dagger}$
\end{tabular}


Since our aim is to investigate the degree of long memory in the returns we further estimate an $\mathrm{AR}(5)$ FIGARCH(1, $d, 1)$ model for all series using the Student$t$ distribution because the return series were found to follow a distribution with heavier tails than the normal, as described previously. We use the quasimaximum likelihood (QML) method to estimate the $\operatorname{AR}(5)$-FIGARCH $(1, d, 1)$ model parameters and employ the BFGS optimization algorithm. All calculations were performed using the econometric package OxMetrics 5.00. Finally, we proceed as in the autoregressive model and compute a number of misspecification tests to investigate the validity of the estimates.

The estimates of the $\operatorname{AR}(5)-\operatorname{FIGARCH}(1, d, 1)$ models for the whole sample period are reported in Tables III and IV. Results show that all coefficients in the variance equation are positive and highly significant at any of the standard levels except for SSE and KLCI indices, where $\widehat{\omega}, \widehat{\alpha}, \widehat{\beta}$ are not significant at $1 \%$ or $5 \%$. Nevertheless, the long memory parameter continues to be significant for all indices indicating that there are long memory effects in the conditional variance for all series as volatility displays little tendency to revert toward its mean. This may imply the violation of the weak form of the efficient market hypothesis as suggested by Elder and Serletis [8] since it demonstrates the potential predictability of a time series.

A more in-depth analysis reveals that the behavior of the $d$ parameter varies in accordance with the degree of development of a market. Accordingly, as illustrated in Fig. 3, the so-called developed markets exhibit a higher level of persistency than the emerging markets. Indeed, the $d$ parameter lies between around 0.39 for SSE returns and around 0.59 for S\&P 500 for the overall sample. The interesting point here is that there is clear evidence of two very distinct groups corresponding to the region. Thus, while all emerging countries show lower levels of long memory, mature economies display a more pronounced degree of memory. We then performed the residual analysis where no evidence of $\mathrm{ARCH}$ effects were found as the ARCH-LM test of Engle [10] and the Ljung-Box test applied to the squared residuals could reject the null of homocedasticity at the $1 \%$ level, which means that the $\operatorname{AR}(5)-F I G A R C H(1, d, 1)$ could capture this feature of the data. As for autocorrelation, we already demonstrated that the autoregressive model was able to remove it from the series and therefore there was no need for additional tests for the residual series of the $\operatorname{AR}(5)-F I G A R C H(1, d, 1)$ model.

TABLE III

FIGARCH $(1, d, 1)$ estimation results for the developed countries returns for the whole sample period. The standard error is presented in parenthesis below each coefficient estimate. $\mathrm{ARCH}-\mathrm{LM}(10)$ and $Q^{2}(10)$ are the Engle [10] test for conditional heteroskedasticity applied to standardized residuals (10 lags) and the empirical statistics of the Ljung-Box test for autocorrelation applied to the squared residuals, with 10 lags, respectively. ${ }^{\dagger}$ significant at the $1 \%$ level, ${ }^{\ddagger}$ significant at the $5 \%$ level.

\begin{tabular}{|c|c|c|c|c|c|}
\hline & S\&P500 & STOXX 50 & FTSE 100 & NIKKEI 225 & HSI \\
\hline \multicolumn{6}{|c|}{ variance equation } \\
\hline$\widehat{\omega}$ & $\begin{array}{c}0.0273^{\dagger} \\
(0.0090)\end{array}$ & $\begin{array}{l}0.03111^{\dagger} \\
(0.0098)\end{array}$ & $\begin{array}{c}0.04698^{\dagger} \\
(0.0155)\end{array}$ & $\begin{array}{c}0.0943^{\dagger} \\
(0.0364)\end{array}$ & $\begin{array}{l}0.04847^{\dagger} \\
(0.0176)\end{array}$ \\
\hline \multirow[t]{2}{*}{$\widehat{\alpha}$} & $0.09226^{\ddagger}$ & $0.14985^{\dagger}$ & $0.15821^{\ddagger}$ & $0.147^{\ddagger}$ & $0.1856^{\dagger}$ \\
\hline & $(0.0448)$ & $(0.0395)$ & $(0.0695)$ & $(0.0737)$ & $(0.0442)$ \\
\hline \multirow[t]{2}{*}{$\widehat{\beta}$} & $0.63426^{\dagger}$ & $0.59037^{\dagger}$ & $0.48035^{\dagger}$ & $0.4908^{\dagger}$ & $0.61538^{\dagger}$ \\
\hline & $(0.1045)$ & $(0.0572)$ & $(0.0914)$ & $(0.1097)$ & $(0.0842)$ \\
\hline \multirow[t]{2}{*}{$\widehat{d}$} & $0.58609^{\dagger}$ & $0.48613^{\dagger}$ & $0.39802^{\dagger}$ & $0.41653^{\dagger}$ & $0.47197^{\dagger}$ \\
\hline & $(0.1060)$ & $(0.0483)$ & $(0.0501)$ & $(0.0687)$ & $(0.0760)$ \\
\hline \multicolumn{6}{|c|}{ diagnostic tests } \\
\hline \multirow{2}{*}{$\begin{array}{c}\mathrm{ARCH}-\mathrm{LM}(10) \\
Q^{2}(10)\end{array}$} & 2.05188 & 0.73926 & 0.71139 & 2.1595 & 1.467 \\
\hline & 47.8821 & 7.55519 & 7.17056 & 13.4095 & 15.0727 \\
\hline
\end{tabular}

The second goal of our research is to evaluate how the long memory parameter behaves over different periods. To this end, we divided the whole sample into four subsamples of three years each, encompassing both stable and crisis periods to test their impact on the memory term; this allows us to evaluate the variation of this parameter across volatile and tranquil periods. Again, we estimate the $\mathrm{AR}(5)$-FIGARCH$(1, d, 1)$ model and compute the diagnostic tests of the standardized residuals. For the sub-sample analysis, we observe that all coefficients are positive and statistically significant at the $1 \%$ or $5 \%$ levels $\mathbb{I}$. The diagnostic tests of the standardized residuals again highlight the suitability of this specification for capturing the heteroskedasticity present in the returns series as given by the non-rejection of the $\mathrm{ARCH}-$ LM test.

Figures 4 to 6 illustrate the evolution of the $d$ parameter over time for all countries. We found three

IWe do not present the results here for space reasons but they will be made available upon request. 
TABLE IV

As Table III, but for emerging countries.

\begin{tabular}{|c|c|c|c|c|c|}
\hline & BUX & WIG & SSE & IDX & KLCI \\
\hline \multicolumn{6}{|c|}{ variance equation } \\
\hline \multirow[t]{2}{*}{$\widehat{\omega}$} & $0.139^{\ddagger}$ & $0.15438^{\dagger}$ & 0.12339 & $0.08661^{\ddagger}$ & 0.03952 \\
\hline & $(0.0541)$ & $(0.0462)$ & $(0.1163)$ & $(0.0438)$ & $(0.0299)$ \\
\hline \multirow[t]{2}{*}{$\widehat{\alpha}$} & $0.43331^{\dagger}$ & $0.22781^{\dagger}$ & 0.15947 & $0.33223^{\ddagger}$ & 0.19631 \\
\hline & $(0.1209)$ & $(0.0555)$ & $(0.2493)$ & $(0.1503)$ & $(0.2107)$ \\
\hline \multirow[t]{2}{*}{$\widehat{\beta}$} & $0.59521^{\dagger}$ & $0.5169^{\dagger}$ & 0.47636 & $0.52189^{\dagger}$ & 0.47814 \\
\hline & $(0.1292)$ & $(0.0728)$ & $(0.3595)$ & $(0.1753)$ & $(0.2683)$ \\
\hline \multirow[t]{2}{*}{$\widehat{d}$} & $0.39312^{\dagger}$ & $0.39041^{\dagger}$ & $0.38731^{\dagger}$ & $0.3899^{\dagger}$ & $0.39717^{\dagger}$ \\
\hline & $(0.0940)$ & $(0.0464)$ & $(0.1294)$ & $(0.0594)$ & $(0.0787)$ \\
\hline \multicolumn{6}{|c|}{ diagnostic tests } \\
\hline$\overline{\mathrm{ARCH}-\mathrm{LM}(10)}$ & 0.44416 & 1.1734 & 0.31715 & 0.54808 & 0.64801 \\
\hline$Q^{2}(10)$ & 4.51573 & 11.3277 & 2.98326 & 5.56805 & 6.12786 \\
\hline
\end{tabular}

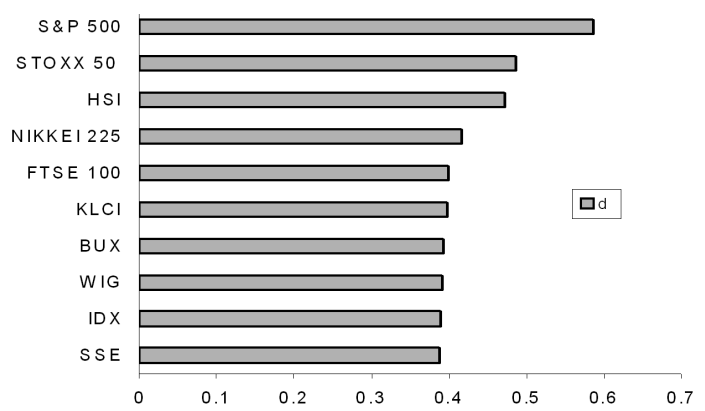

Fig. 3. Estimates of the $d$ coefficient for the full sample period.

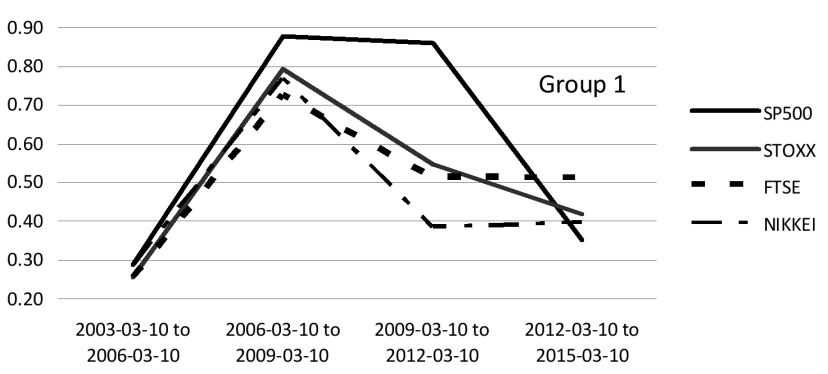

Fig. 4. Estimated $d$ coefficient for S\&P 500, STOXX 50, FTSE 100 and NIKKEI 225 - Group 1.

main patterns. The first group identified - Group 1 - includes the S\&P 500, STOXX 50, FTSE 100 and NIKKEI 225. All these indices experienced a common trend characterized by an unprecedented increase in the memory effect for the period 2006-2009, which may be due to the impact of the 2008 crisis, followed by a decrease in the degree of long memory as a result of the stabilization of market conditions. However, the S\&P 500 still shows very high levels of memory for the sub-period 2009-2012, which may be regarded as a consequence of the sovereign debt crisis.

For the second group (Group 2), which includes the BUX, SSE and IDX indices, the $d$ coefficient generally follows a positive trend over the four sub-samples. Finally, we found that the $d$ coefficient roughly exhibits a constant behavior in the third group (Group 3), which is made up of the HSI, WIG and KLCI indices. These results highlight the different impact of the 2008 and the sovereign debt crises in the variation of the memory parameter for the emerging/developed stock markets. In the first group the memory parameter seems to be seriously affected by these crises exhibiting high levels of long memory; however, the same did not occur for the remaining groups as these crises were typical of the western economies and did not influence emerging markets in the same manner.

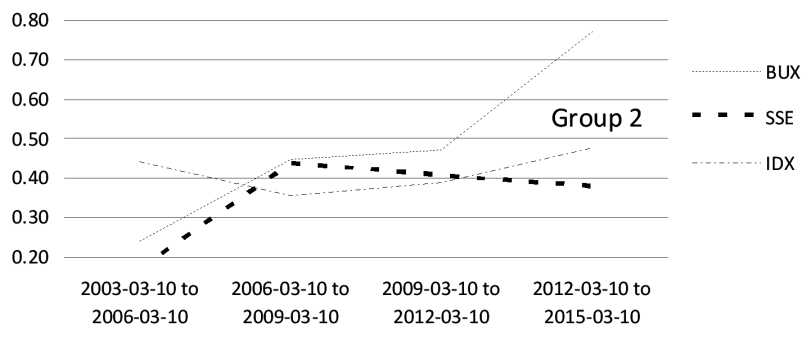

Fig. 5. Estimated $d$ coefficient for BUX, SSE, and IDX - Group 2.

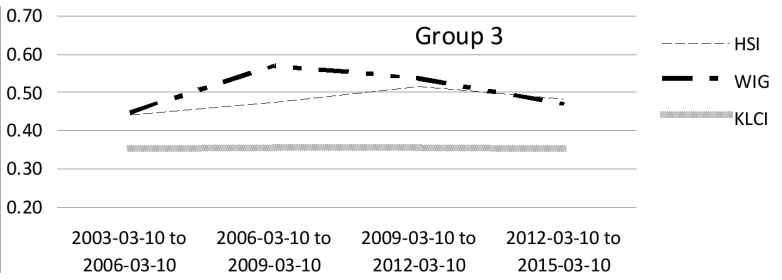

Fig. 6. Estimated $d$ coefficient for HIS, WIG and KLCI - Group 3.

\section{Conclusions}

This paper aimed to analyze the long memory effect in the volatility of ten index returns, namely, S\&P 500, STOXX 50, FTSE 100, NIKKEI 225, HSI, BUX, WIG, 
SSE, IDX and KLCI. Our purpose was twofold: First, we intended to evaluate how this variable behaved across countries in distinct stages of development, namely developed and emerging countries, for the period 2003-2015. Second, we wanted to investigate how long memory varied over time. To this end, we divided the whole sample period into four sub-samples of approximately three years each, encompassing periods of crisis and stabilization in the markets. To conduct our analysis, we employed the $\operatorname{FIGARCH}(p, d, q)$ framework of Baillie et al. [9] in order to account for this property of the data. Its great advantage over previous ARCH-type models is that it embodies both the $\operatorname{GARCH}(p, q)$ and the $\operatorname{IGARCH}(p, q)$ models as special cases. Indeed, when the memory parameter $-d-$ is equal to zero we get the original short memory GARCH, but when $d=1$ we have the infinite memory $\operatorname{IGARCH}(p, q)$ model. In the remaining situations we have $0<d<1$ for an intermediate range of long memory.

Our results for the whole sample period show evidence of long memory for all return series, but the magnitude differs; whereas SSE $(d=0.39)$ is the least persistent market, S\&P $500(d=0.59)$ has the most persistence.

In addition, there is a sharp distinction between the behavior of emerging and developed countries. Next, we refined our analysis and estimated the $\operatorname{FIGARCH}(1, d, 1)$ model for the sub-samples under analysis. This allowed us to diagnose three main trends in the memory parameter evolution corresponding to three distinct groups of countries. The first group encompasses S\&P 500, STOXX 50, FTSE 100 and NIKKEI 225 indices and is generally characterized by an abrupt rise in the $d$ parameter from the first to the second sub-periods and then a fall in the last sub-periods. In contrast, groups 2 (BUX, SSE and IDX) and 3 (HSI, WIG and KLCI) do not seem to be very much affected by the 2008 and 2012 crises. This is not surprising as these are mainly eastern economies that were less influenced by the above-mentioned crises.

In sum, we conclude that long memory is an important characteristic of the data and should be accounted for when addressing market opportunities. However, its influence is not homogeneous and varies in accordance with the country and period under consideration.

\section{References}

[1] H.E. Hurst, Trans. Am. Soc. Civil Eng. 116, 770 (1951).

[2] M.S. Taqqu, V. Teverovsky, W. Willinger, Fractals 3, 785 (1995).

[3] B. Mandelbrot, J.W. van Ness, SIAM Rev. 10, 422 (1968).

[4] N. Vandewalle, M. Ausloos, Physica A 246, 454 (1997).

[5] P. Grau-Carles, Physica A 287, 396 (2000).

[6] P. Oswiecimka, J. Kwapien, S. Drozdz, A.Z. Gorski, R. Rak, Acta Phys. Pol. B 37, 3083 (2006).

[7] L. Calvet, A. Fisher, B.B. Mandelbrot, Cowles Foundation Discussion Paper 1165, (1997).

[8] J. Elder, A. Serletis, Rev. Financ. Econ. 17, 146 (2008).

[9] R.T. Baillie, T. Bollerslev, H.O. Mikkelsen, J. Econom. 74, 3 (1996).

[10] F. Engle, Econometrica 50, 987 (1982).

[11] T. Bollerslev, J. Econom. 31, 307 (1986).

[12] T. Bollerslev, R.Y. Chou, K.F. Kroner, J. Econom. 52, 5 (1992).

[13] R.F. Engle, T. Bollerslev, Econom. Rev. 5, 1 (1986).

[14] T. Bollerslev, J.M. Wooldridge, Econom. Rev. 11, 143 (1992).

[15] J.G. MacKinnon, A.A. Haug, L. Michelis, J. Appl. Econ. 14, 563 (1999). 